\title{
Dynamic localization in walking droplets?
}

\author{
J. MONTES ${ }^{1}$, F. REVUELTA ${ }^{1,2}$ and F. BORONDO ${ }^{2,3, *}$ \\ ${ }^{1}$ Grupo de Sistemas Complejos, Escuela Técnica Superior de Ingeniería Agronómica, Alimentaria y de Biosistemas, \\ Universidad Politécnica de Madrid, Avda. Puerta de Hierro, 2-4, 28040 Madrid, Spain \\ ${ }^{2}$ Instituto de Ciencias Matemáticas (ICMAT), Cantoblanco, 28049 Madrid, Spain \\ ${ }^{3}$ Departamento de Química, Universidad Autónoma de Madrid, Cantoblanco, 28049 Madrid, Spain \\ *Corresponding author. E-mail: f.borondo@uam.es
}

\begin{abstract}
In this paper we explore the possibility of localization in the dynamics of walking droplets on a vertically vibrated liquid surface in the presence of a chaotic external potential and the limit of low memory, i.e., considering the effect of the wave generated only in the last bounce. Taking into account the similarity existing between this macroscopic system and de Broglie's pilot wave theory, this study aims at establishing manifestations of chaos in the latter theory.
\end{abstract}

Keywords. Drops and bubbles; applied fluid mechanics; semiclassical theories and applications.

PACS Nos 47.55.D; 47.85.g; 03.65.Sq

\section{Introduction}

Since the early days of quantum theory its analogy with hydrodynamics was clear. Actually, Madelung [1] showed how by writing the wavefunction in polar form, the time-dependent Schödinger equation can be recast as two coupled ordinary differential equations, one dictating the fate of the probability density, and the other, similar to the Hamilton-Jacobi equation, governing the behavior of the corresponding phase. Later, Bohm [2] used this formalism to define quantum trajectories, in an attempt to solve some of the interpretational difficulties arising in the standard Copenhagen interpretation [3].

A very relevant fact in connection with this is that a decade ago Fort, Couder and coworkers in Paris [4], followed by the group of Bush at MIT [5], showed that a millimetric oil droplet, bouncing on a liquid bath vertically vibrated close (but below) the Faraday wave threshold [6], can self-propel due to the interaction with the waves it generates at the bounces. This phenomenon is shown in the video of Ref. [7]. As can be seen in it, at each impact on the surface the droplet creates a circular capillary wave that in turn excites standing Faraday waves. These waves propagate, damping away with a time that is proportional to the extent to which the amplitude of the forcing vibration approaches the Faraday instability threshold. In this way, one can define a memory parameter $M$ as the damping time over the time interval between successive rebounds. Accordingly, the wave creates a corrugation on the surface which results only from the last $M$ impacts. When $M>>1$, the droplet suffers a small horizontal force, causing it to start "walking" on the liquid surface, and this is the reason why this system is referred to as a walker. Moreover, in this system there is a strong coupling between wave and particle, since the former creates a force for the latter, and the bounces of the latter sustains the former.

The amazing fact is that for high values of the memory, the system exhibits properties that are highly reminiscent of the quantum behavior [8], previously thought to be exclusive of the microscopic realm. In this sense, the fluid can be compared [9] to the quantum pilotwave theories proposed in the early days of quantum mechanics by Louis de Broglie in his double solution conception [10], and further elaborated by Bohm [2].

Indeed, several paradigmatic quantum phenomena have been emulated using bouncing droplets. Diffraction by a single-slit and double-slit interference [11], where the usual quantum pattern [12] is obtained from the angular distribution of droplet trajectories, is one of them. Tunneling [13], where a droplet overcomes the barrier formed by different depths in the liquid container, has also been simulated. Similarly, the existence of orbit quantization-like conditions [14], where the fluid container is subjected to a constant velocity rotating motion and only these droplet orbits corresponding to specific 
values of the radius are stable, has also been reported. Other quantum effects have been reproduced, such as Zeeman-like level splitting $[15,16]$ in experimental situations in which a harmonic potential is implemented in the hydrodynamical system either by means of a ferromagnetic material added to the droplet and an external magnetic field or a rotating container. Also, the emergence of coherent statistics patterns due to interference of the orbits "bouncing" on the boundaries of a closed container [17], which are the macroscopic analogue of the electronic quantum density existing in nanometric corrals on metallic surfaces [18], or entangled bound states consisting of a number of droplets simultaneously performing complicated choreographies [19], has been experimentally observed in this macroscopic system.

One field in which the correspondence between quantum results and the dynamics of classical orbits is very relevant is quantum chaos [20]. By this term, not yet satisfactorily defined [21], one usually refers to the quantum manifestations of chaos in systems that behave classically in a chaotic fashion [22]. One of the most relevant topics in the area of quantum chaos, at least from the semiclassical point of view, is the dynamical localization of the quantum density along unstable periodic orbits caused by a coherent building up of probability due to recurrences [23]. Heller coined the term "scar" for this phenomenon when it happens in eigenstates of classically chaotic systems, such as the Bunimovitch stadium [24]. Recurrences along the homoclinic and heteroclinic circuits associated to periodic orbits [22] have also been shown to contribute to this localization in non-stationary wavepackets $[25,26]$.

If one considers the two main results discussed so far in this section, namely the reminiscence of quantum behavior in the macroscopic walking droplets $[11,13-15,17,19]$ and the quantum chaos localization phenomena [24-26], it seems an interesting idea to investigate if the latter also appears in the former, and this is the aim of the present paper.

For this purpose we have chosen to use an external potential which is classically chaotic, namely the homogeneous quartic two-dimensional oscillator to be defined in the next section, that has been extensively used in many studies of classical and quantum chaos [27]. To investigate the dynamics of the droplets, we will use the simple model of self-propelled drops with the so-called Rayleigh wave force or friction term developed by Labousse and Perrard [28]. Details will be given in the next section.

The organization of the paper is as follows. In the next section we discuss some theoretical aspects of our work, which covers the dynamics of the walking droplets and the external potential function considered. Section 3 is devoted to the description of our results. We present representative trajectories, Poincaré surfaces of sections, attractors and probability density functions. Finally, we conclude the paper with section 4 , where we present a summary of our results and the conclusions.

\section{Theory}

\subsection{Walking droplets}

We assume a fluid of density $\rho$, kinematic viscosity $v$, and surface tension $\sigma$ in a container of depth $H$. In actual experiments this is typically silicon oil. The container is bolted to a vertical vibrating shaker which oscillates sinusoidally with a frequency $\omega=2 \pi f$ and an amplitude $A_{0}$. The effective gravity is then $g+A_{0} \omega^{2} \sin \omega t$ $=g+\gamma \sin \omega t$, where $\gamma$ is a parameter that is controlled in the experiment. Below the Faraday's threshold, $\gamma_{F}$, the bath surface is not excited, and remains at rest despite the fact that it is being shaken vertically. The existing experiments have always being carried out at $\gamma \lesssim \gamma_{F}$.

In this situation, when a small drop impacts vertically on the surface, its unstable equilibrium is perturbed, thus creating two types of waves. The first one is due to the initial impact, and it quickly vanishes. The second one, which is more long-lived, corresponds to the Faraday wave. When the drop hits, for a second time, the now wavy surface of the bath, it impacts on a nondamped away wave, which impulses the drop vertically up. The bouncing, maintaining the wave, can continue repeating itself again and again so that, when stroboscopically illuminated, the particle is seen to levitate. This is made possible since the shaker is adding energy to the droplets. There is another threshold $\gamma_{W}<\gamma_{F}$ at which the droplet stops moving only vertically and it starts walking. All details of these processes are well documented in the existing literature; see, for example, Refs [8, 29,30].

Moreover, in this situation the vertical and horizontal motions of the drop can be assumed to be uncoupled, so that the trajectory of the walker can be studied theoretically in a stroboscopic fashion. The corresponding two-dimensional equation of motion for $X Y$, in which the vertical $Z$ motion is averaged out is given by

$\kappa_{0}(1-\Gamma) \frac{d^{2} \mathbf{x}_{p}(t)}{d t^{2}}+\frac{d \mathbf{x}_{p}(t)}{d t}=-\frac{2}{(1-\Gamma)^{2}} \nabla h\left(\mathbf{x}_{p}(t), t\right)$,

where $\mathbf{x}_{p}(t)=(x(t), y(t))$ gives the position of the particle and $h(\mathbf{x}(t), t)$ represents the height of the surface at a specific point. Also $\kappa_{0}$ is a constant that summarizes all parameters characterizing the fluid, and $0 \leq \Gamma \leq 1$ 
indicates the degree of memory. Furthermore, making a stroboscopic approximation that takes into account all Faraday waves generated up to that point, $h$ results in [31]

$h(\mathbf{x}(t), t)=\int_{-\infty}^{t} J_{0}(|\mathbf{x}(t)-\mathbf{x}(s)|) e^{-(s-t)} d s$.

Considering this expression, we obtain the following integro-differential equation for the motion of the free walkers

$$
\begin{aligned}
\kappa_{0}(1- & \Gamma) \frac{d^{2} \mathbf{x}_{p}(t)}{d t^{2}}+\frac{d \mathbf{x}_{p}(t)}{d t} \\
= & -\frac{2}{(1-\Gamma)^{2}} \int_{-\infty}^{t} J_{1}\left(\left|\mathbf{x}_{p}(t)-\mathbf{x}_{p}(s)\right|\right) \\
& \times \frac{\left(\mathbf{x}_{p}(t)-\mathbf{x}_{p}(s)\right)}{\left|\mathbf{x}_{p}(t)-\mathbf{x}_{p}(s)\right|} e^{-(s-t)} d s .
\end{aligned}
$$

Notice here how the force exerted on the droplet due to the wave profile created by its bouncing is a function of the past history of the droplet (trajectory). Accordingly, this equation shows explicit and quantitatively the dynamical role played by the memory in this theory.

Moreover, one of the fundamental advantages of this formulation is that it allows the inclusion of the force derived from an external potential, $V\left(\mathbf{x}_{p}(t)\right)$, in the following simple way:

$$
\begin{aligned}
\kappa_{0}(1 & -\Gamma) \frac{d^{2} \mathbf{x}_{p}(t)}{d t^{2}}+\frac{d \mathbf{x}_{p}(t)}{d t} \\
& =-\frac{2}{(1-\Gamma)^{2}} \nabla h\left(\mathbf{x}_{p}(t), t\right)-\nabla V\left(\mathbf{x}_{p}(t)\right) .
\end{aligned}
$$

In our study we will consider a simplified version of this expression, valid only in the case of low memory. Actually, we will use the Rayleigh approximation to the wave force/friction developed by Labousse and Perrard in Ref. [28]. These authors considered the case in which the wave is generated only by the last/previous bounce of the droplet. In this case, the height is given by the zero order Bessel function

$h \approx h_{0} J_{0}\left[k_{F}\left|\mathbf{x}(t)-\mathbf{x}\left(t-t_{F}\right)\right|\right]$,

where $h_{0}$ is the amplitude of the field and $k_{F}=2 \pi / \lambda_{F}$ is the vector field of the Faraday wave vector. The associated wave force is related to the local slope of the field, and it can be obtained as the gradient of $h$, which can be further approximated by

$\mathbf{F}_{p}=\gamma_{0} \mathbf{v}\left(1-\frac{v^{2}}{v_{0}^{2}}\right)$, where $\mathbf{v}$ is the droplet velocity vector, $\gamma_{0}=k_{F} T_{F} C / 2$ $-\mu_{0}, v_{0}=\left[16 \gamma_{0} /\left(C h_{0}\left(k_{F} T_{F}\right)^{3}\right]^{1 / 2}, C\right.$ is the proportionality constant between the wave force and $\nabla h$, and $\mu_{0}$ is the apparent friction calculated in [30].

In this way, we finally have the following expression for the motion of the walkers in an external potential

$\frac{d \mathbf{v}}{d t}=-\nabla V(\mathbf{x})-\frac{1}{T_{v}} \mathbf{v}\left(\frac{v^{2}}{v_{0}^{2}}-1\right)$,

$T_{v}=m / \gamma_{0}$ being a characteristic time.

Parameter $v_{0}$ is very relevant in our calculations. It represents the limit velocity of the droplet in the absence of an external potential (as well as for some particular potentials, such as the harmonic one). On the other hand, when an external potential exists, $v_{0}$ gives the average limit velocity. Moreover, $v_{0}$ controls the Rayleigh friction term, in the following way: When the speed of the droplet is bigger than $v_{0}$ it causes a deceleration, while when $v<v_{0}$ and acceleration of the particle takes place.

\subsection{External potential}

In this work we choose to use an external potential which ensures that in the absence of the effects due to the vibrating liquid surface, the trajectories of the walkers are very chaotic. This potential function is to be included in eq. (7). A very popular choice for these types of potentials are chaotic billiards, such as the Bunimovitch stadium, which has been often used both in theoretical and experimental studies [20,22].

In our case, we prefer instead to use the so-called quartic oscillator potential in two dimensions

$V(x, y)=\frac{\alpha}{2} x^{2} y^{2}+\frac{\beta}{4}\left(x^{4}+y^{4}\right)$,

for a number of reasons. First, it is a continuous, smooth potential easy to be treated by numerical procedures. Second, this potential is homogeneous and then in the Hamiltonian case, the trajectories exhibit mechanical similarity. That is, the classical motion at any value of the energy can be obtained by scaling the results from $E=1$. This avoids the hassles derived from the dependence of the phase space structure with the energy. Third, using this potential avoids the complications (discontinuities) due to the bouncing of trajectories at hard walls (although in the case of the droplet's paths, this is alleviated by the effect of the wave also bouncing at the walls). Fourth, the dynamical behavior of this system is extremely chaotic, and free of the marginally stable (vertical and whispering gallery) motions [22] that complicate the dynamics of the Bunimovitch stadium. And 
fifth, this potential has been extensively used in different studies of classical and quantum chaos [27].

At this point it should be remarked that, although creating such a potential in an actual experimental setup it is not an easy task, it is however not impossible either, since it can be obtained, at least to some extent, by adequately shaping the bottom of the fluid container or using suitably shaped magnetic fields and a ferromagnetic fluid.

The quartic potential (8) includes two parameters $\alpha$ and $\beta$ whose values determine the degree of chaoticity. For $\alpha=1$ and $\beta=1 / 100$ the corresponding trajectories present a very high degree of chaoticity. Actually, for a long time it was thought to be totally ergodic (in the limit $\beta \rightarrow 0$ ) until Dahlquist and Russberg found a family of tiny islands of regularity [32]. As the value of $\alpha$ is increased, the potential becomes more and more regular. Indeed, for especial cases, such as $\alpha=0$ or $\alpha=\beta=1$, the behavior is completely regular.

In the top part of figure 1 we present the quartic potential (8) in the form of contour plots for two sets of values of the parameters.

To ascertain the dynamical character (regular or chaotic) of the corresponding classical trajectories we use Poincaré surfaces of section (PSOS) [22]. The method consists of representing the intersections of a swarm of trajectories all propagated at the same energy with a given surface in phase space. In our case we have chosen $E=1$ and $C:=\left\{x=0, P_{x}>0\right\}$. The results are shown in the bottom part of figure 1. As can be seen, for the lower value of $\alpha$, the dynamics are more
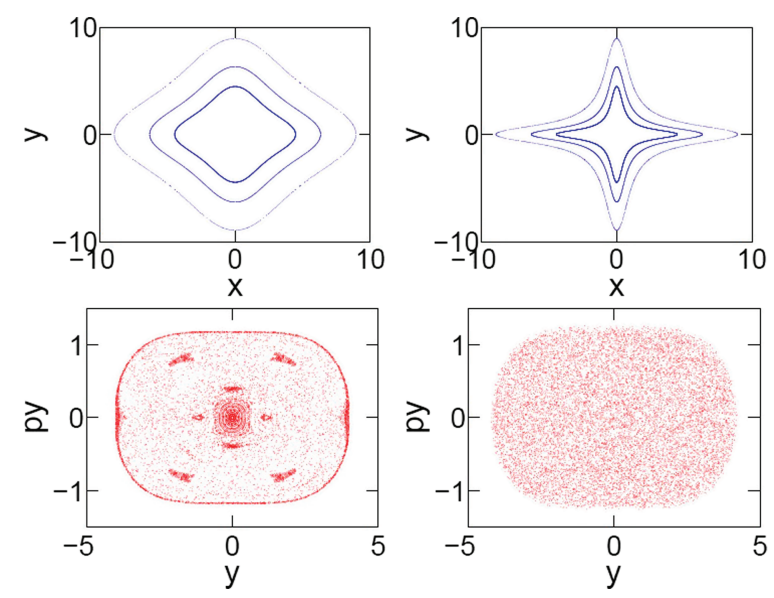

Figure 1. (Top) Potential energy function (8) used in our calculations for $\beta=1 / 100$, and $\alpha=1 / 20$ (left) and $\alpha=1$ (right). Three equipotential curves, for $V=1$ (innermost), 4, and 16 (outermost), are shown in each panel. (Bottom) Corresponding composite Poincaré surfaces of sections using $\mathcal{C}:=\left\{x=0, P_{x}>0\right\}$ as the sectioning plane for $E=1$ (see text for details). regular, and small islands of regularity are seen inside the widespread sea of chaoticity. On the contrary, for $\alpha=1$ the full available phase space is (apparently, recall [32]) all filled with chaos.

In this work, we use the first set of potential parameters, i.e. $\beta=1 / 100$ and $\alpha=1 / 20$, corresponding to the more regular dynamics, since here the analysis of the effect of the pilot wave on the trajectories is easier.

\section{Results and Discussion}

\subsection{Trajectories}

In this section we present our results for droplet trajectories computed by using the potential function (8), numerically integrating eq. (7) for different values of the involved parameter $T_{v}$.

We show in figure 2 a typical trajectory for the quartic oscillator with $\beta=1 / 100$ and $\alpha=1 / 20$ (recall that in this case the free trajectories show some degree of regularity; see left part of figure 1) in the frictionless case, i.e. $T_{v}=\infty$, and in the small friction limit, $T_{v}=0.9$, both computed for the same initial conditions and a value of $v_{0}=1$. (This value will be used in all calculations throughout the paper unless otherwise stated.)

As can be seen, in the case of figure $2 \mathrm{a}$ and $\mathrm{c}$, where no memory effect of the bounces is taken into account since $T_{v}=\infty$, the trajectory is totally chaotic. This is apparent in the views considered, namely the plot of the absolute value of the potential energy vs. position $(x, y)$ in figure $2 \mathrm{a}$, and the plot of the temporal evolution of the kinetic energy $E_{c}$ in figure 2c. However, when some memory is introduced by setting $T_{v}=0.9$, which is within the small memory limit, the trajectory
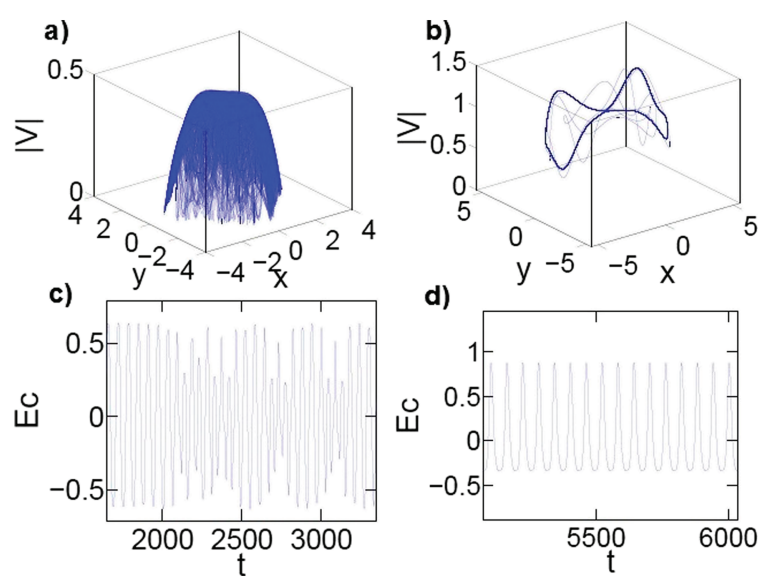

Figure 2. Two typical trajectories for the quartic oscillator with $\beta=1 / 100, \alpha=1 / 20$ and $v_{0}=1$ in the: (a) case of no friction, i.e. $T_{v}=\infty$, and (b) small friction limit for $T_{v}=0.9$. (c-d) Time-evolution of the corresponding kinetic energies. 
(after a short transient period not shown in the plot) gets stabilized into a periodic motion, despite the fact that such regions are not common in the corresponding Hamiltonian case (see PSOS in figure $1 \mathrm{left}$ ). Actually, it is the effect of the pilot wave which causes such stabilization. The conclusion from the results in this figure is very clear: Parameter $T_{v}$ is very relevant in our study, and exploring which droplet dynamical behaviors can be found when it is varied is certainly worthwhile.

In figure 3 we show the results of such exploration. In it, we have plotted some characteristic trajectories for the quartic oscillator with $\beta=1 / 100$ and $\alpha=1 / 20$ for increasing values of $T_{v}$ (from top to bottom); recall that its inverse value controls the strength of the Rayleigh friction term. Each horizontal row consists of two examples computed at the same value of $T_{v}$ but with different initial conditions. As can be seen, the dynamical character of the trajectories is very sensitive to the value of $T_{v}$, being more chaotic for the smaller values of the parameter and more regular for the higher values, as should be expected $a$ priori. Moreover, for the same values of $T_{v}$ the different trajectories that are obtained are much more similar, even if they stabilize into different periodic motions which are equivalent by symmetry (as are the cases shown in figure 3).

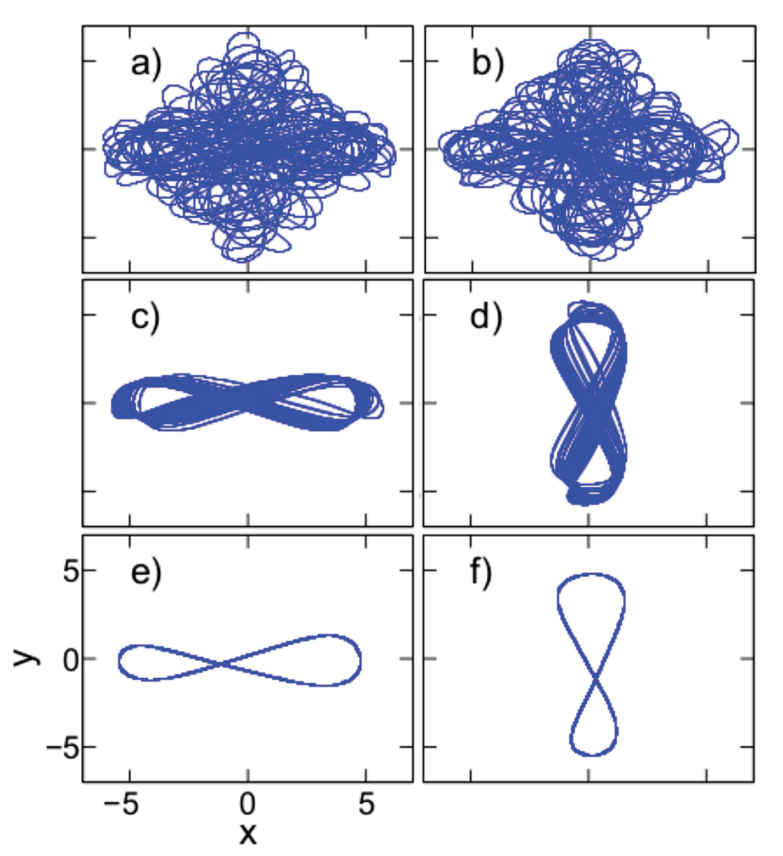

Figure 3. Some characteristic trajectories for the quartic oscillator with $\beta=1 / 100$ and $\alpha=1 / 20$ for two different initial conditions, and: $(\mathbf{a}-\mathbf{b}) T_{v}=0.2,(\mathbf{c}-\mathbf{d}) T_{v}=0.4$, and (e-f) $T_{v}=0.5$.

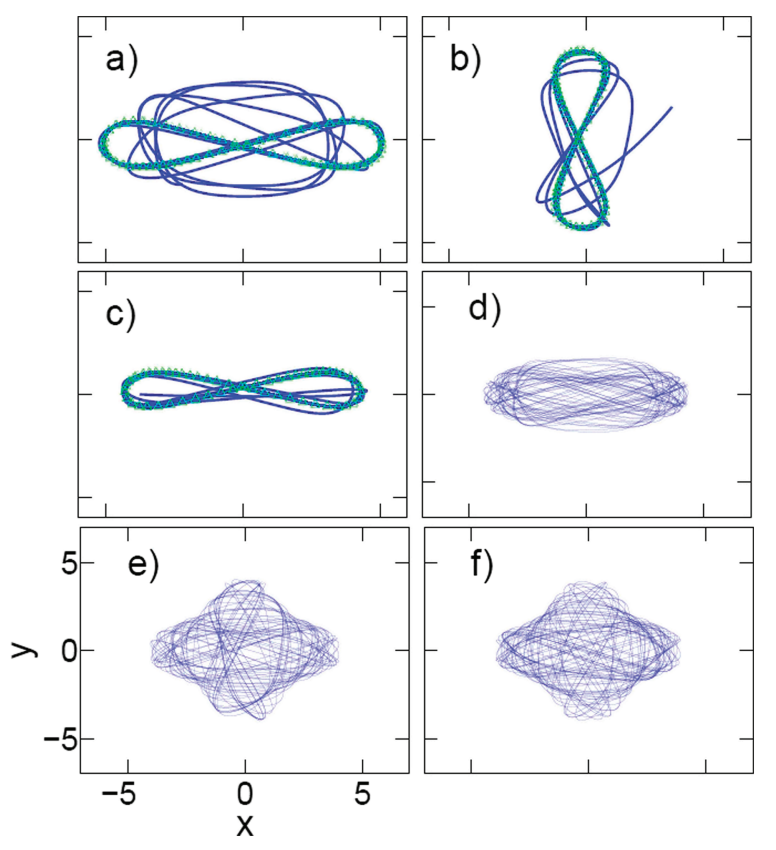

Figure 4. Same as figure 3 for a single initial condition and different values of the memory parameter $T_{v}$ : (a) $T_{v}=0.7$, (b) $T_{v}=0.9$, (c) $T_{v}=1.0$, (d) $T_{v}=1.1$, (e) $T_{v}=1.8$, and (f) $T_{v}=2.1$. In this plot we represent the transient part of the trajectory in blue, and the part of it in the final attractor in green.

To further investigate the sensibility of the dynamical character of the trajectories to the value of $T_{v}$, we present some more cases in figure 4. In it, we represent some trajectories for higher values of $T_{v}$, separately marking, in blue and green respectively, the transient and final, i.e. attractor, parts of it. As can be seen, the attractor presents a shape similar to a rounded bow tie or figure eight. Also in the first three cases the final attractor is essentially either the same (putting aside the small differences due to the differences in $T_{v}$ ) or equivalent by symmetry. However, when the value of $T_{v}$ is further increased (less effect of the friction term) above $T_{v}=1.0$ the trajectories all become chaotic, without stabilizing in time into any recognizable attractor. These results confirm again the sensitivity of the dynamical character of the trajectories to the strength of the friction term.

We continue our exploration in figure 5, where more representative trajectories corresponding to higher values (lower friction) of the parameter $T_{v}$ are presented. As can be seen, the same conclusions are here also valid, and more interestingly a new, more complex attractor periodic motion appears. In this case, the shape of the motion in configuration space resembles that of those paper clips designed to hold a large number of paper sheets. Actually, this shape is also found as a periodic orbit in the Hamiltonian quartic oscillator [33]. 


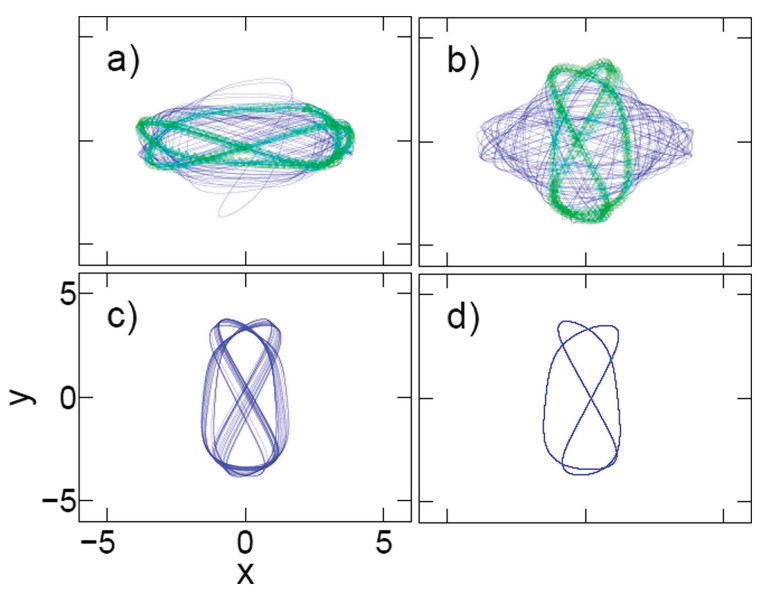

Figure 5. Same as figure 4 for: (a) $T_{v}=2.3$, (b) $T_{v}=2.4$, (c) $T_{v}=2.5$, and (d) $T_{v}=2.7$.

\subsection{Poincaré surfaces of section}

To complete the discussion of figures $3-5$, we consider now the corresponding composite PSOS, as defined in subsection 2.2. As indicated there, this tool is not only ideal to ascertain the dynamical character of the walker's trajectories, but it also provides at the same time a global picture of the dynamics in the whole phase space.

Some results are shown in figure 6 for low values of the friction parameter corresponding to $T_{v}=0.2-2.7$. These figures were produced with results from 50 trajectories started with random initial conditions uniformly covering the available phase space, each one

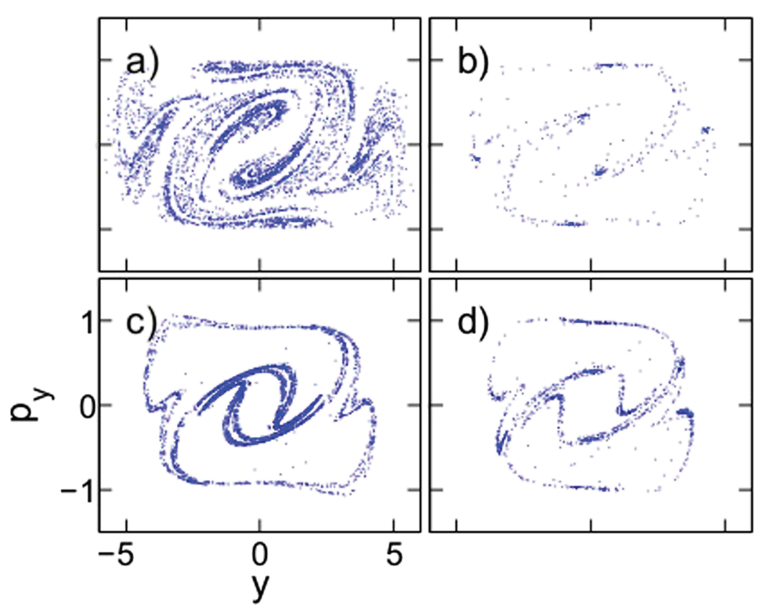

Figure 6. Composite Poincaré surfaces of section for different values of the memory parameter: (a) $T_{v}=0.2$, (b) $T_{v}$ $=0.9$, (c) $T_{v}=1.1$, and (d) $T_{v}=2.7$. Each plot consists of the results generated from 50 trajectories propagated until they crossed 200 times each the surface of section, $\mathcal{C}$. See text for details. being propagated until it crossed 200 times the SOS, $c$. The different panels show the dynamical effect of the attractor, which "sucks" the trajectories starting near the origin $\left(y_{0}, P_{0}\right) \simeq(0,0)$, and also at $\left(y_{0}, P_{0}\right)$ $\simeq( \pm 2.5, \pm 0.5)$, leaving those regions almost empty. Actually, the effect is more pronounced as $T_{v}$ increases, and for $T_{v}=1.1$ and 2.7 the whirlpool shape of the attractor is clearly visible. When examined in detail, we see that for $T_{v}=0.2$ [panel (a)] the effect of the attractor is small, being most points dispersed all over the available phase space. They correspond to the transient. However, all trajectories finally concentrate around six points located approximately at $\left(y, P_{y}\right)=( \pm 4.1, \pm 0.09)$, $( \pm 0.5, \pm 0.99)$, and $( \pm 0.3, \pm 1.2)$. Notice the inversion symmetry with respect to the origin of these points indicated by the " \pm " signs. For $T_{v}=0.9$ it is clear that the effect of the attractor is much more pronounced, the transient part of the trajectory being much more reduced, and the concentration on the final points indicated before much more clearly defined. As $T_{v}$ increases to a value of 1.1 , the attractor evolves to a quasiperiodic trajectory that leaves, after the transient, a clear whirlpool shape in the central part of the PSOS. Finally, this structure begins to slowly disintegrate at $T_{v}=2.7$.

To conclude this part of the discussion, we present in figure 7 similar results, but for much larger values of $T_{v}$. As can be seen here, the effect of the friction is much lower, and we start to recover the free trajectory results

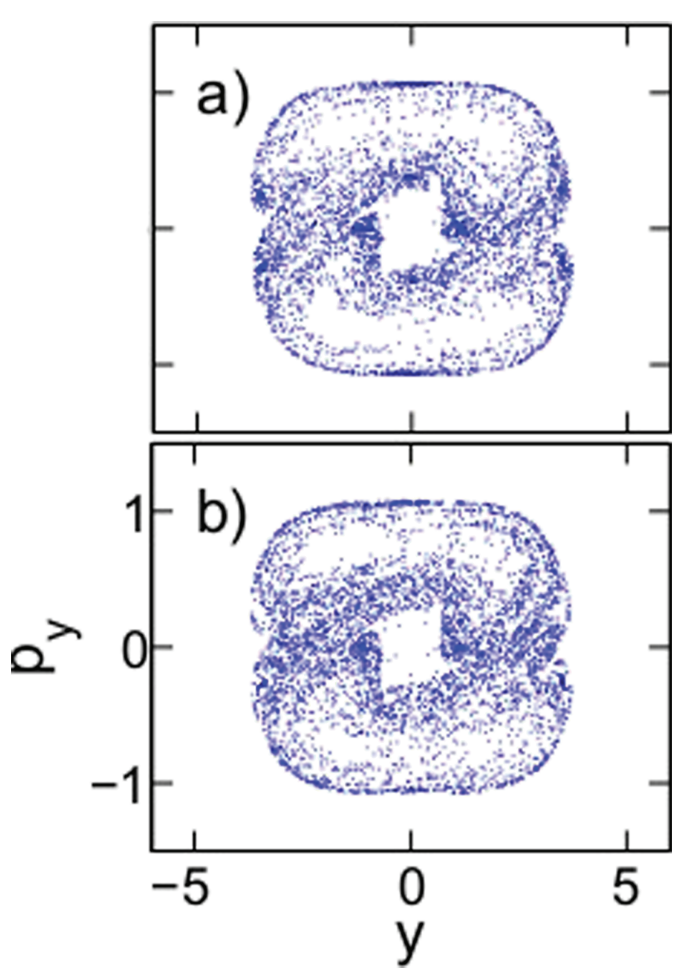

Figure 7. Same as figure 6 for $(\mathbf{a}) T_{v}=20$ and (b) $T_{v}=25$. 
shown in the left panels of figure 2, which correspond to a very chaotic dynamics, as discussed in subsection 2.2.

\subsection{Attractors}

In this section we explore the existence of attractors in our system.

For this purpose, we propagate for a sufficiently large number of time steps a delocalized trajectory for $T_{v}=0.3$ and $v_{0}=0.4$, which is subsequently deconstructed by splitting it into different time intervals. The results are shown in figure 8 . We have chosen to present them in the form of a projection into the phase space plane $\left(x, v_{x}\right)$. As can be seen in figure 8a, the complete trajectory appears totally delocalized, filling all the space except for a tiny region near the origin. However, when split in different fragments (figure 8b-d) the trajectory is seen to get temporarily trapped, or localized, in different regions of the phase space, successively jumping among them. For example, in figure $8 b$, we present the results for the interval $n=43000-45000$ which is seen to consist of a first part oscillating in the inner region near the origin (in green), and a second part moving in the outer region (in blue). This second part is shown more clearly in figure $8 \mathrm{c}$. Finally, in figure $8 \mathrm{~d}$, we show how this behavior repeats for longer times in the dynamics of our trajectory. These results clearly indicate that there exist two dynamically separated regions in the corresponding phase space.

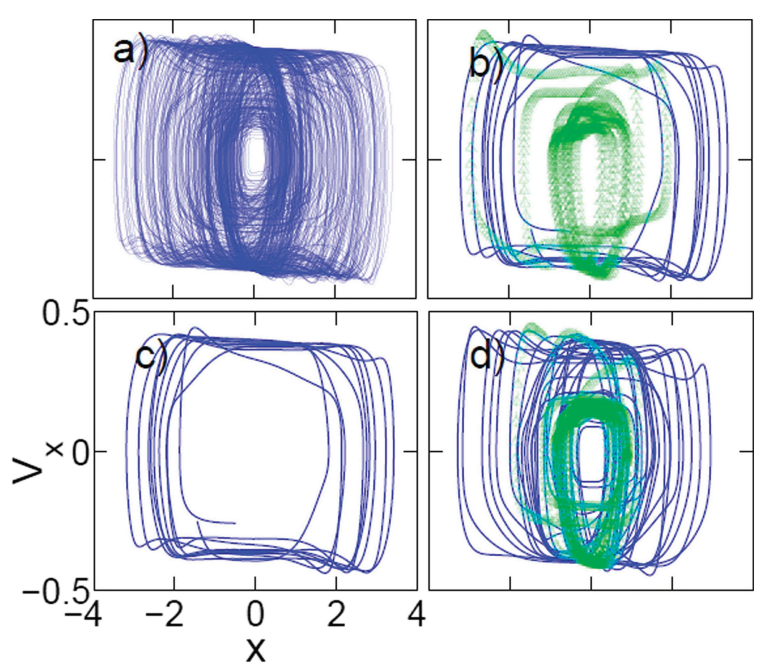

Figure 8. Structure of the attractor for $T_{v}=0.3$ and $v_{0}=0.4$ obtained by deconstruction of a representative trajectory inside it. A projection into the phase space plane $\left(x, v_{x}\right)$ is used. Time intervals: (a) complete trajectory, (b) trajectory steps 43000-45000 in green and 45000-48000 in blue, (c) trajectory steps 45000-48000, (d) trajectory steps 56000-64000 in blue and 64000-66000 in green.

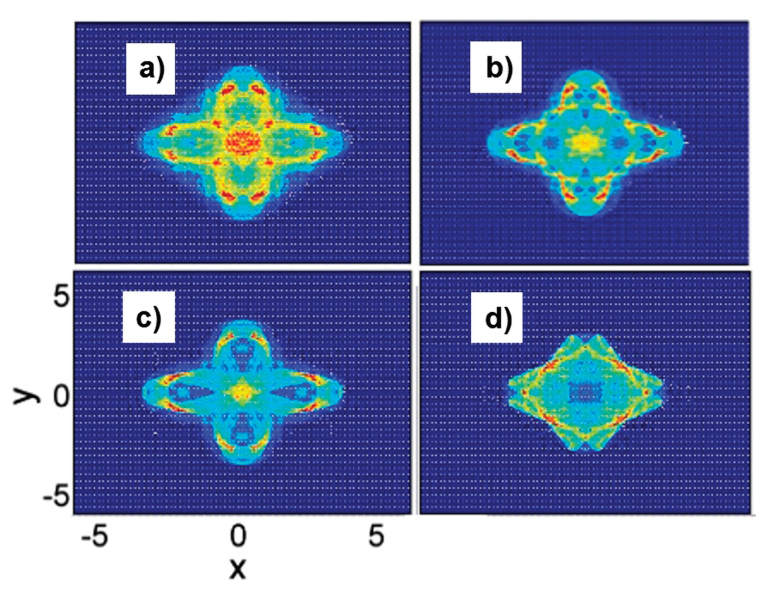

Figure 9. Probability density function for different values of $T_{v}$ : (a) 0.28 , (b) 0.52, (c) 0.56, and (d) 2.5, in a color scale. Red color indicates the maximum and dark blue the minimum.

\subsection{Probability density function}

To conclude the Results section we discuss the computation of probability density functions (PDF), similar to what was performed in Ref. [17] by Bush et al. to obtain results that could be compared to the experimental quantum mechanical probabilities in electron corrals existing in the literature [18]. The procedure is quite easy. It simply consists in dividing the configuration space into small square bins, and counting the number of times that a given trajectory passes through every bin. The corresponding value of the counting is represented by a different color in the associated pixel of the plot. In this way a histogram is obtained, representing the probability density function associated to the trajectory, which can be suitably represented either in 3D or in a color scale, as we have chosen in the present work.

The results corresponding to four non-localized trajectories propagated at different values of $T_{v}$ are shown in figure 9. As can be seen, in all cases the PDF appears localized along periodic orbits of the system, despite the fact that the associated trajectories from which they are obtained do not. In figure $9 \mathrm{a}-\mathrm{c}$ the localization takes place on the figure of eight motion described in figures 3 and 4, while in figure 9d an accumulation on the so-called square box type periodic orbit (see Ref. [33]) is seen.

\section{Summary and conclusions}

In this paper, we have presented a theoretical study on the dynamic of walking droplets bouncing off the surface of a liquid bath in a container which is vertically vibrated just below the Faraday threshold and 
is subjected to an external potential function described by a homogeneous quartic $2 \mathrm{D}$ polynomial.

We have found that despite the chaotic character of the forces induced by the applied potential, the trajectories of the walkers very often get stabilized on periodic motions due to the effect of the friction induced by the capilary waves created by the bounces. Our study has been carried out in the low memory limit, where only the effect of the last bounce is taken into account, thus assuming that the effect of previous bounces has died away.

Examining the topology of the trajectories and the corresponding Poincaré surfaces of section we have assessed the dynamical nature of our system. Moreover, the existence of attractors in the dynamics has also been explored.

Finally, we have computed and analyzed the probability density function arising from non-localized trajectories, and found that this magnitude seems to localize along periodic orbits of the system, similar to what happens with the scarring phenomena on some eigenstates of classically chaotic Hamiltonian systems described in the field of quantum chaos.

\section{Acknowledgements}

The research leading to these results has received funding from the Ministerio de Economía y Competitividad under Contracts MTM2015-63914-P and ICMAT Severo Ochoa under SEV-2015-0554.

\section{References}

[1] F Madelung, Z. Phys. 40, 322 (1926)

[2] D Bohm, Phys. Rev. 85, 166 (1952); ibid. 180 (1952)

[3] J von Neumann, Mathematical Foundations of Quantum Mechanics (Princeton University Press, Princeton, 1955)

[4] Y Couder, S Protiere, E Fort and A Boudaoud, Nature 437, 208 (2005)

[5] J W M Bush, Phys. Today 47 (August, 2015)

[6] M Faraday, Phil. Trans. Royal Soc. (London), 121, 299 (1831)

[7] https://www.youtube.com/watch?v=nmC0ygr08tE

[8] J W M Bush, Annu. Rev. Fluid Mech. 47, 269 (2015)

[9] D M Harris and J W M Bush, Phys. Fluids 25, 091112 (2013)

[10] L de Broglie, Comptes Rendues 177, 507 (1923); and Une Interprétation Causale et Nonlinéaire de la Mechanique Ondulatoire: La Théorie de la Double Solution (Gauthiers-Villars, Paris, 1956)

[11] Y Couder and E Fort, Phys. Rev. Lett. 97, 154101 (2006)
[12] R P Feynman, R B Leighton and M Sands, The Feynman Lectures on Physics (Addison-Wesley, New York, 1964)

[13] A Eddi, E Fort, F Moisy and Y Couder, Phys. Rev. Lett. 102, 240401 (2009)

[14] E Fort, A Eddi, A Boudaoud, J Moukhtar and Y Couder, Proc Nat. Acad. Sci. USA 107, 17515 (2010); A U Oza, D M Harris, R R Rosales and J W M Bush, J. Fluid Mech. 744, 4045 (2014); A U Oza, O Wind-Willassen, D M Harris, R R Rosales and J W M Bush, Phys. Fluids, 26, 082101 (2014)

[15] S Perrard, M Labousse, M Miskin, E Fort and Y Couder, Nat. Commun. 5, 3219 (2014); M Labousse and S Perrard, Phys. Rev. E 90, 022913 (2014)

[16] A U Oza, A Trajectory Equation for Walking Droplets: Hydrodynamics Pilot-Wave Theory, MIT Ph.D. Thesis (2014)

[17] D M Harris, J Moukhtar, E Fort, Y Couder and J W M Bush, Phys. Rev. E 88, 011001(R) (2013)

[18] M F Crommie, C P Lutz and D M Eigler, Science 262, 218 (1993)

[19] S Protiere, S Bohn and Y Couder, Phys. Rev. E 78, 036204 (2008); C Borghesi, J Moukhtar, M Labousse, A Eddi, E Fort and Y Couder, Phys. Rev. E 90, 063017 (2014)

[20] M C Gutzwiller, Chaos in Classical and Quantum Mechanics (Springer-Verlag, New-York, 1990); P Cvitanovic, R Artuso, R Mainieri, G Tanner and G. Vattay, Chaos: Classical and Quantum (Niels Bohr Institute, Copenhagen, 2016)

[21] M V Berry, Physica Scripta 40, 335 (1989)

[22] A J Lichtenberg and M A Lieberman, Regular and Chaotic Dynamics (Springer Verlag, New York, 1992)

[23] E J Heller, in Chaos and Quantum Physics, edited by M J Giannoni, A Voros and J Zinn-Justin, Les Houches session 52 (North Holland, Amsterdam, 1989)

[24] E J Heller, Phys. Rev. Lett 53, 1515 (1984)

[25] G G de Polavieja, F Borondo and R M Benito, Phys. Rev. Lett. 73, 1613 (1994)

[26] D A Wisniacki, E G Vergini, R M Benito and F. Borondo, Phys. Rev. Lett. 94, 054101 (2005); ibid., 97, 094101 (2006)

[27] O Bohigas, S Tomsovic and D Ullmo, Phys. Rep. 223, 43 (1993)

[28] M Labousse and S Perrard, Phys. Rev. E 90, 022913 (2014)

[29] S Protiere, A Boudaoud and Y Couder, J. Fluid. Mech. 554, 85 (2006)

[30] J Molacek and J W M Bush, J. Fluid. Mech. 727, 582 (2013); ibid. 612 (2013)

[31] A U Oza, R R Rosales and J W M Bush, J. Fluid. Mech. 737, 552 (2013)

[32] P Dahlquist and G Russberg, Phys. Rev. Lett. 65, 2837 (1990)

[33] F Revuelta, E Vergini, R M Benito and F Borondo, Phys. Rev. E 87, 042921 (2013) 